\title{
Citizen Electronic Identities using TPM 2.0
}

\author{
Thomas Nyman \\ Aalto University and \\ University of Helsinki \\ thomas.nyman@aalto.fi
}

\author{
Jan-Erik Ekberg \\ Trustonic \\ jan-erik.ekberg@trustonic.com
}

\author{
N. Asokan \\ Aalto University and \\ University of Helsinki \\ asokan@acm.org
}

\begin{abstract}
Electronic Identification (eID) is becoming commonplace in several European countries. eID is typically used to authenticate to government e-services, but is also used for other services, such as public transit, e-banking, and physical security access control. Typical eID tokens take the form of physical smart cards, but successes in merging eID into phone operator SIM cards show that eID tokens integrated into a personal device can offer better usability compared to standalone tokens. At the same time, trusted hardware that enables secure storage and isolated processing of sensitive data have become commonplace both on $\mathrm{PC}$ platforms as well as mobile devices.

Some time ago, the Trusted Computing Group (TCG) released the version 2.0 of the Trusted Platform Module (TPM) specification. We propose an eID architecture based on the new, rich authorization model introduced in the TCGs TPM 2.0. The goal of the design is to improve the overall security and usability compared to traditional smart card-based solutions. We also provide, to the best our knowledge, the first accessible description of the TPM 2.0 authorization model.
\end{abstract}

\section{Categories and Subject Descriptors}

K.6.5 [Management of Computing and Information Systems]: Security and Protection

\section{INTRODUCTION}

Identification and authentication of citizens using Electronic Identification (eID) mechanisms is beginning to be deployed in several European countries. In some countries, such as Estonia, eID tokens are already used widely, while eID use is taking hold in other countries such as Belgium, Austria and Spain [12]. The typical use of eID is to access government services such as filing tax returns, accessing healthcare records or digitally signing applications for state benefits. However, since eID tokens provide strong identity

This work is based on an earlier work: Citizen Electronic Identities using TPM 2.0, to appear in the Proceedings of the 4th international workshop on Trustworthy embedded devices, TrustED'14, November 3, 2014, Scottsdale, Arizona, USA, http://dx.doi.org/10.1145/2666141.2666146 verification, they are also used for other services, both public and private. For example in Estonia, more than 100000 persons use their eID cards as a form of public transport tickets [12]. They are also used as substitutes for driving licenses and e-banking tokens, as well as physical access control tokens for libraries and swimming pools [13]. Furthermore, personal computer operating systems allow for seamless integration of PKCS\#15 cards which makes it possible to use them with common applications such as e-mail clients with S/MIME support.

Identity tokens, referred to as "Electronic Signature Products" (ESPs) by the relevant EU directives, must be trustworthy. ESPs are logically isolated security tokens that use a signature key to digitally sign a request on behalf of a user and thereby prove the user identity. The ESPs are under the control of users, and can be carried by them or be integrated into their devices. The ESP is, however, not necessarily a physical smart card - so far, the ESP function has also been successfully integrated into operator SIM cards ${ }^{1,2}$, as well as in network services controlled by password-based access. With the increased usage of personal user devices, integrating an eID mechanism securely also in these devices will help improve usability of such eID compared to standalone eID tokens.

Given the centrality of the role eID architectures will play in the lives of people, it is important to identify and ensure the security, usability and regulatory requirements an eID architecture should meet in order to be deemed trustworthy. There are two major requirements that need to be met. First, the link between the natural person represented by an eID and the person using that eID must be established. Legislation typically provides exact regulation on the required issuance procedure for physical identity tokens. Since an eID is to be interchangeable with a physical one, the same laws need to be applied to eID issuance with little or no modification [13]. Second, the eID credentials must be logically isolated from other software and must be used only when the person associated with the eID intends it. The latter requirement is met by subjecting the use of the eID credential to user authentication, usually based on PINs. PINs protecting eID credentials are subject to a number of policy restrictions intended to balance security and usability [7]. For instance, the eID issuer or a service provider relying on eID may stipulate the type of protection (e.g. PIN) for the credential. Also, since a personal device can hold multiple

\footnotetext{
${ }^{1}$ Estonian Mobile-ID: http://e-estonia.com/component/mobile-id/

${ }^{2}$ Finnish Mobile Certificate: http://www.mobiilivarmenne.fi/en/
} 
eID-related credentials, it must be possible to associate the same PIN with multiple credentials.

The Trusted Computing Group (TCG) ${ }^{3}$ is the leading organization specifying standards for trusted hardware on a wide range of devices ranging from servers and personal computers to mobile devices. Hundreds of millions of Trusted Platform Modules (TPMs) are deployed in such devices. The new version of the TPM specification ${ }^{4}$ includes a new, rich authorization model for specifying flexible access control policies for objects protected by the TPM. This makes it possible to support the kind of complex policies required for protecting eID credentials on the range of devices traditionally equipped with TPMs. TPM 2.0 specifications have been public for some time. However, as far as we are aware, there has been no accessible explanation of the TPM 2.0 authorization model.

In this paper, we describe how an eID architecture can be designed and implemented using TPM 2.0. In particular, we make the following contributions:

- We provide the first accessible description of the TPM 2.0 authorization model. We believe that this will help researchers understand the power of the model and to design other security and privacy solutions based on it.

- We describe the detailed design of an eID architecture based on TPM 2.0. We show how this solution improves the overall security and usability compared to eID solutions based on traditional physical tokens such as removable smart cards.

- We identify possible enhancements to the TPM 2.0 authorization model. If adopted, these enhancements would further improve the flexilibility of the authorization model, and greatly simplify the definition of certain kinds of policies.

The paper is organized as follows: Section 2 introduces necessary background on current European eID solutions and TPMs. In particular, Section 2.3.4 describes the TPM 2.0 authorization model in detail. Section 3 introduces the requirements for our design, while the design itself is presented in Section 4. An analysis of the design is given in Section 5. Section 6 suggests some potential improvements to the current TPM 2.0 authorization model. Related work is discussed in Section 7. Section 8 concludes.

\section{BACKGROUND}

\section{1 eID in Europe}

The legal status of eID mechanisms in Europe is based on the EU signature directive from 1999 (1999/93/EC) and the data protection and privacy directives from 1995 (95/46/EC) and 2002 (2002/58/EC) [11]. These directives guarantee legal equivalence between digital signatures done with the citizen cards and physical signatures by the associated person. They set the privacy framework for how identity information can be stored and managed in servers interacting with identified citizens.

Technically, the current European ID cards are often (contactless) ISO 7816 smart cards, corresponding to the

\footnotetext{
${ }^{3}$ http://www.trustedcomputinggroup.org/

${ }^{4}$ http://www.trustedcomputinggroup.org/media_room/news/352
}

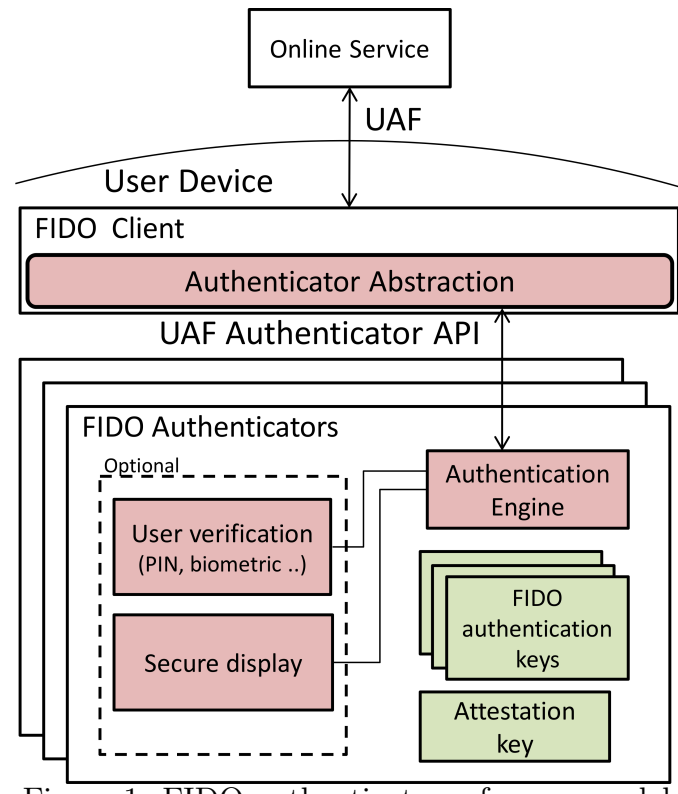

Figure 1: FIDO authenticator reference model

PKCS\#15 interface standard of which there are jurisdictionspecific profiles. Most identity cards contain at least two signature keys and provide two forms of signatures - the legally binding "qualified" signature key for document signing is separated from the "authentication" keys to be used in more interactive settings. Key usage authorization is governed with PINs.

Such identity tokens come with a number of drawbacks that influence large-scale deployment; the physical token must be present for each authentication, and all devices must be equipped with compatible card readers. As most smart cards lack user interfaces, if credential authorization requires a PIN, users typically have to enter it via a potentially untrusted device.

Nevertheless, in the EU, the main challenges hindering the adoption of an interoperable pan-European eID scheme are considered to be not only technological, but also legal. In a recent report by the eID team in the European Commission's Institute for Prospective Technological Studies (IPTS) [2], a series of principles that aim at providing the legal underpinning for a future eID legal scheme are elaborated on. These include, among others, the principles of usercentricity, anonymity and pseudonimity and the principles of multiple identities, identity portability and un-linkability.

\subsection{FIDO Alliance}

The Fast Identity Online (FIDO) alliance ${ }^{5}$ is an industry consortium whose mission is to reduce the reliance on passwords for user authentication in internet transactions. The alliance has the backing of many industry giants, and may turn out to be a significant reference model even for government eID applications.

The FIDO specifications define authentication architectures and protocols based on strong authentication and biometrics [10]. The Universal Authentication Framework (UAF) protocol enables online services to support passwordless and multi-factor security. In fact, a typical eID credential is very close to the reference authenticator component in the FIDO architecture (see Figure 1). The credential can

\footnotetext{
$\overline{{ }^{5} \text { https://fidoalliance.org/specifications }}$
} 
(and should) have support for device-local user authentication for credential enablement, that can take the form of a PIN, password or e.g. a biometric.

However, what sets the FIDO protocols apart from many legacy eID setups, is that FIDO clients generate serviceprovider specific asymmetric key pairs for authentication. Using a different key pair with every service provider improves user privacy, since it protects against linking the activity of a user in cases where service providers collude. Supporting many dynamically allocatable authentication keys is not technically difficult to implement in TPMs, or even with smart cards. This is a practical requirement also for government eID. In such a set up, it is desirable to bind several key pairs to the same instance of device-local user authentication.

For mobile devices, and for the use cases discussed in this paper, the FIDO token primarily operates as a First Factor Bound Authenticator [9]. In this mode of operation, the primary means of authentication is strengthened by the authenticator being bound to an identifiable / attestable device. In the FIDO architecture, a credential can also be removable, either as a First Factor Roaming Authenticator, or augmenting another authentication as a Second Factor Authenticator.

\subsection{TPMs}

TPMs are system components that provide a hardwarebased approach for secure non-volatile (NV) storage, cryptographic key generation and use, sealed storage and (remote) attestation. The primary scope of a TPM is to assure the integrity of a platform by providing means to identify (and report on) the hardware and software components that comprise the platform. The notion of "trust" in the context of TPMs stems from the expectation of behaviour that can be determined from this identity. The state of the TPM is separate from the state of the platform on which it reports, and the only way for the host system to interact with a TPM is through an interface defined in the TPM specifications $[16$, 17]. TPMs differentiate themselves from conventional secure cryptoprocessors in that they provide platform binding, i.e. proof of an association between a cryptographically verifiable identity and the platform itself.

TPMs can be implemented as single-chip components with separate physical resources dedicated to the TPM. The first TPM microcontrollers became available in $2005^{6}$, and since then TPMs are included in many new laptop computers, primarily in business product lines. However, the notion of a platform in the context of TPMs is not tied PCs, nor to a particular operating system. Hardware-based Trusted Execution Environments [5] (TEEs) have become commonplace in modern mobile platforms. They provide secure, integrityprotected processing environments, consisting of processing, memory and storage capabilities, that are isolated from the regular processing environment, sometimes called the rich execution environment (REE), where the device operating system and applications run. As TEEs provide the necessary capabilities for housing TPMs, firmware-based TPMs are a more likely deployment model on mobile devices.

\subsubsection{Attestation}

\footnotetext{
${ }^{6}$ Infineon press release announcing the SLB 9635 TPM 1.2: http://www.infineon.com/cms/en/corporate/press/news/releases / 2005/132443.html
}

Compared to smart cards, the issuance of secure functionality to a TEE in a mobile device out in the field will require an extra set of trust anchors. This particular problem setting is described in a whitepaper on User-Centric Provisioning [1] published by the GlobalPlatform association $^{7}$. Compared to physical smart cards, which as a rule are provisioned by a trustworthy manufacturer on behalf of the token issuer, a TPM as a TEE function or application is manufactured and put to market in a trust domain that normally does not a priori include any relation to the service issuer. Thus, the connection between the token issuer trust domain and the TEE must be established at service provisioning time.

At large this problem is one of attestation, i.e. the service issuing entity must both authenticate the targeted endpoint as well as convince itself of the current setup, version and revocation status of the TEE deployed in that endpoint. Furthermore, for data provisioning the issuer must confirm that the endpoint within the TEE, i.e. the Trusted Application (TA), will be the issued service and not some other code running in the TEE.

In physical TPMs, the attestation property is embodied by Endorsement Keys (EKs) - unique device secret keys that are associated with a device certificate via a trusted party, like the TPM manufacturer. In the TPM 1.2 specification the endorsement key is a decryption key which, using a trustworthy protocol to a "privacy CA", can certify locally generated "Attestation Identity Keys" (AIKs) - signature keys used in the actual attestation events. In the TPM 2.0 specification the endorsement key can also be a signature key. With physical TPM chips, the generation of the EK credential can take place during chip manufacturing, and the credential is best stored in the non-volatile memory of the TPM chip for later use. This approach needs no attestation external to the TPM, since the manufacturing process can be secured to a needed level. On the other hand, for post-manufacturing deployment of firmware TPMs, relevant attestation needs to be provided by the underlying platform. In this paper we assume that the TPM 2.0 (and its EK) can be properly attested by an underlying functionality, and therefore the eID service provider, whether being the government or a private entity, can trust the TPM they are interacting with as the device-specific, fully isolated security component that it is defined to be.

\subsubsection{PCRs}

Platform Configuration Registers (PCRs) are integrityprotected registers in TPMs that are used to store aggregate measurements regarding the security state of the system. A PCR value is a representation of the state of a particular (software) environment. Each PCR holds a digest value consisting of an accumulative hash of previous PCR values. Apart from resetting the PCR to an initial value, the only way to modify a PCR value is to extend a measurement value into the PCR. When a measurement is extended into a PCR, the new digest value is calculated as follows:

$$
P C R_{\text {new }}=\mathbf{H}\left(P C R_{\text {old }} \| \text { digest }\right)
$$

where

$$
\begin{aligned}
& P C R_{\text {new }} \\
& P C R_{\text {old }}
\end{aligned}
$$

\footnotetext{
${ }^{7}$ http://www.globalplatform.org/
} 
$\mathbf{H}()$

digest

is the hash function associated with the PCR

is the measurement value extended into the $\mathrm{PCR}$

As a result of the old digest value being hashed into the new one, any deviation in a reported sequence of events causes an irrevocable change in the eventual PCR digest value. In other words, the PCR digest value is unique for the specific order and combination of digest values that have been extended into a particular PCR. The state of a system, represented by a set of PCRs, can be used in attestation (see Section 2.3.1).

\subsubsection{TPM 1.2 Authorization}

In the TPM 1.2 specification [16], access to TPM operations and objects secured by the TPM (e.g. cryptographic keys) are protected via an authorization mechanism. Access to such an object is obtained via the proof of knowledge of a shared secret associated with the object. In TPM parlance, this shared secret is known as the AuthData of the object. Henceforth, we refer to this shared secret as the owner authorization value, as in general, knowledge of the AuthData is treated as complete proof of ownership of a protected object or operation, with the exception of asymmetric keys locked to a set of particular PCR values during their creation (as discussed below). The TPM places no additional requirements on the use of the object. An overview of the TPM 1.2 authorization model is shown in Figure 2a.

In order to securely pass proof-of-knowledge of the owner authorization value to the TPM, three protocols are used; the Object-Independent Authorization Protocol (OIAP), the Object-Specific Authorization Protocol (OSAP), and the Delegate-Specific Authorization Protocol (DSAP). These protocols allow the caller to establish a confidential authorization session with the TPM. Depending on the protocol used, the authorization session has different properties, e.g. the OIAP protocol allows access to multiple protected objects to be authorized during the same authorization session, with the limitation that OIAP sessions cannot be used for key creation, or other operations that would introduce new authorization information to the TPM. OSAP sessions, on the other hand can only be used manipulate a single object, but allows new authorization information to be transmitted to the TPM. DSAP provides support for delegating access to an object without disclosing its owner authorization value. Instead, object owners may specify a set of operations on an object, which are authorized via an delegation authorization value, provided by the caller as the authData instead of the owner authorization value when operations on an object are invoked using delegated privileges. For further information on the TPM 1.2 authorization protocols, we refer the reader to Appendix A.

In addition, as mentioned earlier, cryptographic keys may be locked to particular PCR values upon key creation. If this is the case, the key is only usable as long as certain PCRs have the particular values associated with the locked key. In this way, PCR values can be used to ensure that certain keys are accessible only to authorized software. This is typically used in combination with the DSAP delegation mechanism to allow a trusted process access to a protected object without user intervention. DSAP checks for the continued validity of such PCR selections, and any change to the PCR values causes the invalidation of the DSAP session. The combination of the delegate authorization value and PCR selections gives the TPM 1.2 four distinct authoriza-

\begin{tabular}{|c|c|c|c|}
\hline case & authValue & PCR selection & authorization method \\
\hline (1) & secret & yes & password and \\
(2) & secret & no & platform state \\
(3) & well-known & yes & platform state \\
(4) & well-known & no & - \\
\hline
\end{tabular}

Table 1: TPM 1.2 authorization modes

tion modes, listed in Table 1 . When a PCR selection is set, the delegation authorization value associated with the delegated key may be a fixed, well-known, value (case (3)). This is because, if the trusted process is to execute automatically, the only way for the trusted process to store the delegation authorization value would be to seal ${ }^{8}$ it against the process's PCR measurement values, but as the delegation mechanism already checks the PCR selections, the verification of the delegate authorization value is redundant.

\subsubsection{TPM 2.0 Enhanced Authorization}

In the TPM 2.0 specification [17], objects stored within the TPM have an authValue property associated with them. The authValue is directly comparable to the TPM 1.2 AuthData, and may be used as a password for object authorization. In addition, the TPM 2.0 specification introduces Enhanced Authorization (EA) policies, which supersede the TPM 1.2 authorization mechanisms and allows object owners and administrators to require specific assertions or actions to take place before access to a protected object is allowed. The policy associated with an object may be arbitrarily complex, even though internally the policy is reduced to a single statistically unique digest value known as the authPolicy. The authPolicy is associated with the TPM entities the corresponding policy applies to. An overview of the TPM 2.0 enhanced authorization model is shown in Figure 2b.

Access to all objects making use of enhanced authorization takes place via a session-based authorization procedure. In order to access a TPM object, the caller initiates a policy session with the TPM. Subsequently the caller issues a sequence of policy commands to the TPM.

Each policy command in the authorization policy is an assertion that a particular statement is true in order for the policy to be satisfied. For instance, a particular policy might require that certain PCRs have specific values in order for access to a TPM object to be authorized. As a side-effect of a true policy assertion, each such policy command modifies a digest value associated with the session, characteristic of the particular policy expressed via the sequence of policy commands. This running accumulation of the digest value is called the policyDigest. When a policy session is started, the associated policyDigest is initialized to zero. Then, as each assertion corresponding to a certain policy command is evaluated, the policyDigest is updated in a manner similar to PCR extension:

policyDigest $_{\text {new }}=\mathbf{H}\left(\right.$ policyDigest $_{\text {old }} \|$ commandCode $\|$ commandArgs $)$ where

policyDigest $_{n e w} \quad$ is the new policy session digest value policyDigest $_{\text {old }} \quad$ is the previous policy session digest value

${ }^{8}$ Sealing data against some PCR values refers to the act of encrypting the data in such a way that the TPM will later decrypt it only if the PCRs have the given values. This may be used to ensure that the data can be opened only if the platform is in a known and trusted state. 


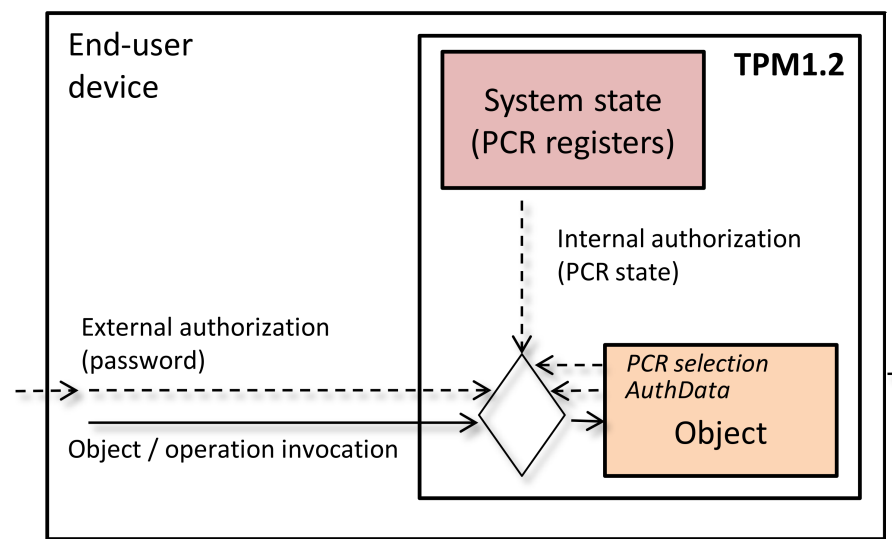

(a) TPM 1.2 authorization model

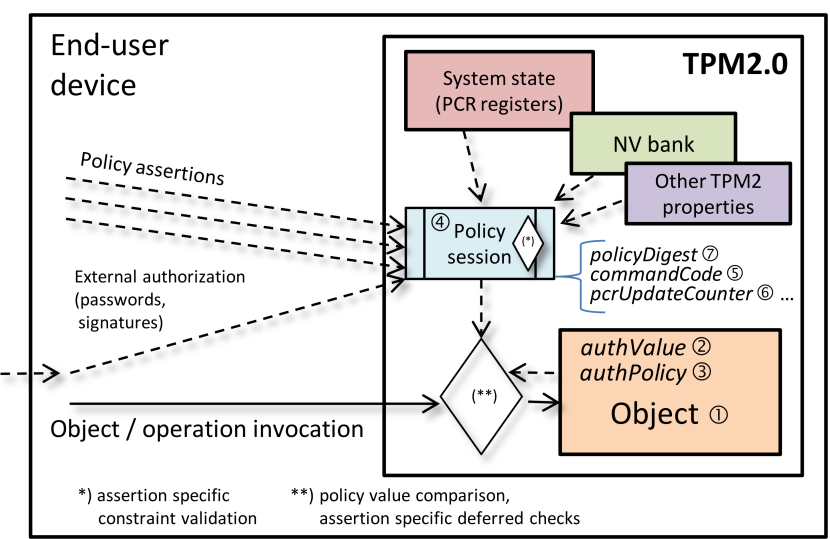

(b) TPM 2.0 enhanced authorization model

Figure 2: Overview of TPM authorization models

\begin{tabular}{|ll|l|}
\hline \multicolumn{1}{|c|}{ Item } & \multicolumn{1}{c|}{ Description } \\
\hline (1) & Object & Key or data stored within the TPM \\
(2) & Object $\rightarrow$ authValue & Byte string used as password in access authorization \\
(3) & Object $\rightarrow$ authPolicy & Digest value used for policy session access authorization \\
(4) policySession & Authorization used for gaining access to an Object by satisfying an \\
& & associated policy \\
(5) policySession $\rightarrow$ commandCode & Command code for TPM command being authorized \\
(6) policySession $\rightarrow$ pcrUpdateCounter & Stored PCR update counter reading \\
(7) policySession $\rightarrow$ policyDigest & Digest value calculated as a result of policy command invocation \\
& & during a policy session \\
\hline
\end{tabular}

Table 2: Glossary of TPM 2.0 Enhanced Authorization Terminology

$\mathbf{H}() \quad$ is the hash function used to update the policyDigest

commandCode is a value which identifies the policy command commandArgs are dependent on the condition asserted

Some policy commands also have the ability to reset the policyDigest value. This occurs conditionally with regard to the previous policyDigest value. We will see that this construct enables not only branching policies, but also policies to be changed based on signatures generated by an external authorization entity:

\section{if condition then}

$$
\text { policyDigest }_{\text {new }}=\mathbf{H}(0 \| \text { commandCode } \| \text { commandArgs })
$$

Only policy commands modify the policyDigest; other TPM 2.0 commands do not. Later in this section, we provide selected examples of different types of policy commands. For descriptions of other commands referred to elsewhere in this paper, we refer the reader to Appendix B.

Finally, after the policy command sequence has been completed, the final value of the policyDigest for the session is compared to the authPolicy of the object being accessed. A match indicates that the sequence of invoked policy commands matches, and satisfies the assertions expressed by the policy, successfully completing the authorization. The authPolicy value associated with an object upon creation can either be calculated in software, or in a special trial policy session, during which all assertions are assumed to succeed and the policyDigest can be retrieved from the TPM at the end of the session.

\section{Types of Policy Assertions.}

TPM 2.0 EA policy commands fall into three categories: immediate, deferred, and combined assertions. In this section, we will provide examples and discuss the specifics of each of the three policy assertion types.

Immediate assertions are policy commands which only affect the policyDigest. An example of an immediate policy assertion is the TPM2_PolicyNV() command, which asserts an arithmetic comparison between an input value and a value stored in a specified non-volatile storage element. If the condition holds, the policyDigest is updated accordingly, otherwise it remains unchanged. Specifically:

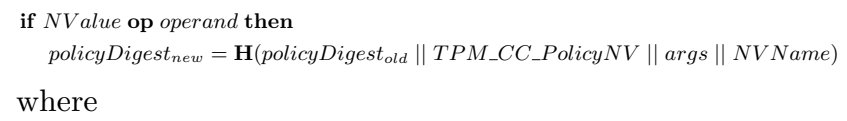

Deferred assertions unconditionally update the policyDigest based on input values and record specific constraints in the context of the current policy session. When the session is used to make the final authorization decision, the stored constraints are validated at that time. For instance, the TPM2_PolicyCommandCode() policy command is used to verify that the policy session is only used to authorize a particular command. This is achieved by storing the command code for the command being authorized in the current session context. The policyDigest is updated unconditionally, namely:

policyDigest $_{\text {new }}=\mathbf{H}\left(\right.$ policyDigest $_{\text {old }} \|$ TPM_CC_PolicyCommandCode $\|$ code $)$ 
where code is the command code for the TPM command being authorized. When the final authorization decision is made, the TPM will verify that the command used to operate on the object being authorized is in fact the command identified by the command code stored in the session context.

Combined assertions validate a precondition regarding the TPM state, and record some parameters in the current policy session context used for deferred checks later on. An example is the TPM2_PolicyPCR() command, which can be used to validate that a specified PCR has the expected value. If the caller provides an expected value, the value of the specified PCR is compared immediately to the expected value; if the values match, the policyDigest is updated accordingly:

policyDigest $_{n e w}=\mathbf{H}\left(\right.$ policyDigest $_{\text {old }} \| T P M_{-} C_{C} C_{-}$PolicyPCR $\|$PCRs $\|$digest $)$

where

PCRs is a bit mask corresponding to the PCR selection

digest is the hash of the PCR values in the selection

If the caller does not provide an expected value, the policyDigest is updated as indicated above, but the validity of the PCR values will not be known until the policy session is used for authorization (i.e. when the policyDigest is compared to the authPolicy). However, merely verifying the PCR value as part of a precondition leaves the authorization policy susceptible to Time-Of-Check Time-Of$U_{s e}^{9}$ (TOCTOU) race conditions in cases where PCR values have changed in the interval between the invocation of the TPM2_PolicyPCR() command, and the time the actual authorization decision is made. To avoid TOCTOU conditions, the TPM keeps track of PCR changes by incrementing a monotonically increasing counter, the pcrUpdateCounter, each time a PCR is updated. When TPM2_PolicyPCR() is invoked, the current value of the PCR update counter is stored in the current session context. If an expected value was provided, the PCR update counter is updated only if the immediate PCR value check succeeds. On subsequent TPM2_PolicyPCR() invocations, and when the policy session is used for authorization, the value of the PCR update counter is compared against the stored counter; the authorization will fail unless the counter values match.

\section{Policy $O R$, and AND.}

As mentioned in Section 2.3.4 branching policies are made possible by resetting the policyDigest value as part of the successful assertion. This disrupts the running digest value, allowing subsequent assertions to proceed from an independent, yet well-known, value. The logical disjunction is embodied by the TPM2_PolicyOR() command. When invoked, TPM2_PolicyOR() is passed a list of digest values, each corresponding to a digest value that is accepted as a valid precondition for a successful TPM2_PolicyOR(). The policyDigest is updated conditionally only if its current value is in this list:

$$
\begin{aligned}
& \text { if } \text { policySession }_{\rightarrow} \text { policyDigest in } \text { digest }_{1} \ldots \text { digest }_{n} \text { then } \\
& \text { policyDigest }_{n e w}=\mathbf{H}(0 \| \text { TPM_CC_PolicyOR } \| \text { digests })
\end{aligned}
$$

where

digest $_{1} \ldots$ digest $_{n}$ is a list of valid digest values ${ }^{10}$

\footnotetext{
${ }^{9}$ CWE-367: Time-of-check Time-of-use Race Condition:
} http://cwe.mitre.org/data/definitions/367.html digests $\quad$ is the concatenation digest $_{1}\|\ldots\|$ digest $_{n}$

The reasoning behind this scheme is that $\mathbf{H}\left(\right.$ digest $_{1}\|\ldots\|$ digest $\left._{n}\right)$ is a well-known, fixed value. For an arbitrary digest digest $_{1} \ldots$ digigest $\left._{n}\right\}$, it is computationally infeasible, due to the properties of the hash function $\mathbf{H}$, to find a concatenation digest $_{x}\|\ldots\|$ digest $^{\prime}\|\ldots\|$ digest $_{z}$ such that:

$\mathbf{H}\left(\right.$ digest $_{1}\|\ldots\|$ digest $\left._{n}\right)=\mathbf{H}\left(\right.$ digest $_{x}\|\ldots\|$ digest $^{\prime}\|\ldots\|$ digest $\left._{z}\right)$

Although the set of EA policy commands does not include an explicit logical AND operation, the way the policyDigest is updated by policy commands, each new value being dependent on the previous one, not only acts as an implicit logical conjunction, but also imposes an order dependence on the sequence of consecutive policy commands.

\section{External Authorization.}

While some EA policies within the TPM may be altered by changing the corresponding authPolicy value, policies associated with key objects and NV memory elements may not. However, it is often the case that static access control policies are "brittle" in the sense that they cannot accommodate for changes in the system that makes the policy essentially unusable. This applies for instance to a policy that seals a piece of data to a set of fixed PCR values to ensure that the data is only accessible when the system has been booted in a specific configuration. In this case, the PCR values could correspond to measurements of the BIOS and operating system kernel. If there is a BIOS update by the Original Equipment Manufacturer (OEM), the measurement value in the corresponding PCR will change, rendering the data inaccessible because the policy cannot accommodate for the BIOS update in advance. These kinds of situations require flexible policies that may be modified in an indirect way after-the-fact. For such cases, the TPM 2.0 EA mechanism provides the TPM2_PolicyAuthorize() command.

The TPM2_PolicyAuthorize() command asserts that the current policyDigest is authorized by an external entity via the signing of the corresponding digest value and an optional policy classifier ${ }^{11}$. If the policyDigest is authorized in such a way, it is reset, and replaced by the name of the signing key used for authorization. If present, the policy classifier can act as a nonce to limit the use of the signature key, as the classifier is extended to the policyDigest:

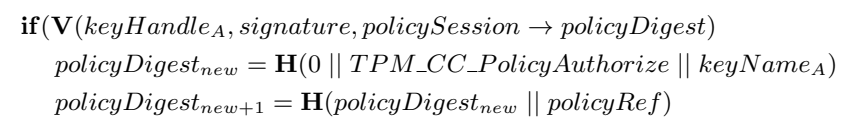
where

$A$ is the authorization key is the signature verification algorithm for $A$ keyHandle $_{A}$ is the handle for $A$ signature is the signature of the policy to be authorized keyName $_{A}$ is the name of the object corresponding to $A$ policyRef is the policy classifier

\footnotetext{
${ }^{10}$ We note that the TPM 2.0 specification limits the number of digests to $n=8$. However, by nesting multiple TPM2_PolicyOR() operations, the effective size of the list can be expanded indefinitely.
} 
The public portion of key objects stored by the TPM is represented in the name of the object. In the case of asymmetric keys, this means that the new policyDigest value is derived, in part, from the public key of the authorizing entity. If a symmetric key is used, its public portion is derived by hashing the key material together with obfuscation values which prevents the public portion from leaking information about the sensitive key material. In other words, any key can be used for the external authorization, but each individual key will leave a matching trace in the policyDigest. Therefore that binding will constitute the identification of the key needed for the authorization.

Returning to our sealing example, the policy that seals the data can be made robust with regards to software updates via a TPM2_PolicyAuthorize() that occurs after the PCR measurements have been extended into the policyDigest with TPM2_PolicyPCR( $)^{12}$. This allows the OEM to provide a signature for $\mathrm{PCR}$ values corresponding to the new BIOS. With either authorized set of of PCR values, the final policyDigest value will be the same, even though the intermediate values after TPM2_PolicyPCR() differ.

\section{REQUIREMENTS}

The requirements for an eID token can be divided into functional, security, and privacy requirements. Some translate into platform requirements, others are restricted to the token itself or apply to token interaction. An overview of eID requirements is shown below:

1. Functional requirements:

(a) Binding the identity of a physical person to multiple identity keys stored on personal device

i. One-to-many relationship between PIN and keys

ii. PIN and PUK with limited number of tries

(b) API compatibility with smart card-based system

2. Security requirements:

(a) Cryptographic requirements

(b) Confidentiality of identity key

(c) Code isolation of operations on the key against software and other keys stored on the device

\section{Privacy requirements:}

(a) Prevention of unauthorized access to credential

(b) Linkability control

\subsection{Functional requirements}

The Application Programming Interface (API) to eID tokens resident in mobile devices must be as similar as possible to the API of existing smart card-based ID systems (Req. 1b), i.e. a PKCS\#15-based interface, with a 3-attempt

\footnotetext{
${ }^{11}$ In practice, the signature is verified by the TPM in a separate TPM2_VerifySignature () command invocation, which produces a ticket that is subsequently passed to TPM2_PolicyAuthorize to, if valid, provide proof that the TPM has validated the signature using a particular key. The reason for this seems to be purely a performance optimization, as it is more efficient to verify the ticket than it would be to load a TPM object each time authorization occurs to verify the signature. ${ }^{12}$ In this case, the expected PCR values would not be provided by the caller in the TPM2_PolicyPCR() invocation.
}

PIN and a PUK for enabling key signatures (Req. 1(a)ii) is the baseline in such an ecosystem. For TPM-based eID, a compatibility layer to provide the necessary API interoperability is needed.

Application requirements for eID sometimes also support and refine the general security requirements. In discussions with representatives of our local jurisdiction (personal correspondence, Feb 28, 2014) [7], it became clear that there is a wish to address the binding problem in mobile phone based tokens by having multiple client keys, both in parallel and over time (Req. 1a). This leads to a requirement that PIN policy and PIN handling are linked to keys only by association, e.g. using PIN values that are shared among many keys and changeable independently from the key objects they are associated with (Req. 1(a)i).

\subsection{Security requirements}

Security requirements include minimum token key strength [8], and the use of modern hash algorithms in identity certificates. For most jurisdictions the minimum eID requirement today is equivalent to 2048 bit RSA and SHA256 for the certificate signature. In this paper, we do not address these requirements directly; for our purposes we assume that the underlying TPM 2.0 implementation supports the necessary primitives.

The requirements on the token platform have been summarized by Dimitrienko et al. [4]. These are the confidentiality of the identity key and the code isolation for the identity integrity verification algorithm operating on the identity key. The isolation must hold against software in the device, but also with regards to other credentials that may be stored and used in the same token. The access control to the credential, with respect to unauthorized users as well as unauthorized code (e.g. malware) in the device must be guaranteed. Last, the integrity of the credential code must be ascertained. Often it is also necessary to ascertain the origin or integrity of the calling code as well.

Certification servers are subject to stringent security evaluation and processes, and certificate enrolment is also rightly considered an important security function, since it cements the proof of the binding between the physical person and his identity token. Reliable attestation is therefore paramount to proper enrolment (see Section 2.3.1).

\subsection{Privacy requirements}

Privacy requirements for eID [14] include preventing unauthorized access to the identity token and applying linkability control either in the eID client or at a server. Mechanisms to achieve the latter includes providing identity assertions rather than full identity information as part of eID transactions, and, where applicable, keeping digital citizen identity numbers separate from conventional national identification numbers, e.g. social security numbers. One way to achieve this is the use of service-provider specific asymmetric keys, as in the FIDO protocols. Protocol confidentiality, i.e. using the identity only in the context of a secure channel, safeguards against eavesdropping.

\section{DESIGN}

\subsection{Architecture}

Our design addresses the security demands for an eID token, as outlined in Section 3. We operate in an system 


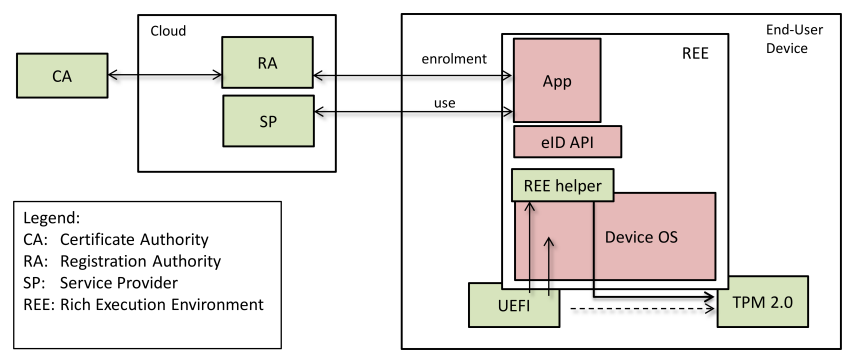

(a) System architecture

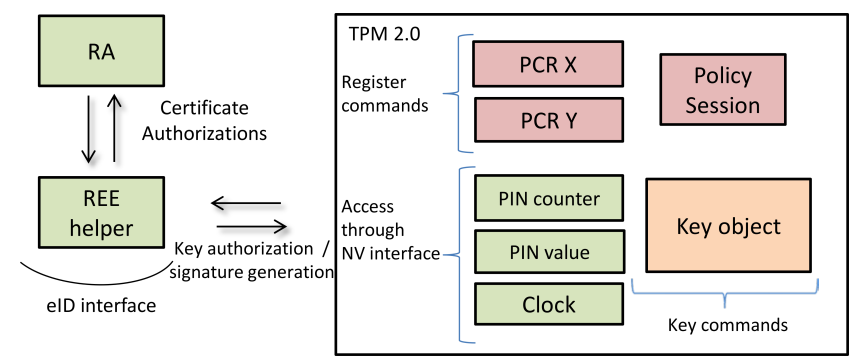

(b) Device internal architecture

Figure 3: eID architecture

architecture illustrated in Figure 3a. The device contains a TPM 2.0. According to specification, this module operates in isolation from the (REE) operating system, and has its own non-volatile storage, random number source and needed cryptographic primitives. The implementation of the TPM 2.0 module can be separate hardware, or, especially in mobile devices, it can be implemented inside a TEE. For the purposes of this paper, the chosen implementation path is irrelevant.

According to TPM specifications, the end-user device must provide a Core Root of Trust for Measurement (CRTM) that supplies basic platform software integrity measurements to the TPM in a reliable manner. This function is provided for the TPM by the platform's authenticated or secure boot-up code, and is in Figure 3a represented by the Unified Extensible Firmware Interface (UEFI) secure boot [19]. For the purpose of the eID example, reliable measurements of a) the OS and b) a component we call $R E E$ helper need to be provided to the TPM. The version of the OS (and e.g. its TPM drivers) and the REE helper that will run our TPM policy represents the part of the software stack that serves as a precondition for the TPM 2.0-based eID system to work. We expect an authorization of the fact that necessary components are in place and that e.g. user PINs can be entered by the user at some level of assurance. We will see that the integrity of the REE components are not otherwise a requirement for the policy or the eID function to work correctly.

Our eID system also contains off-device components. The Registration Authority (RA) is the network facing entity that provides credential enrolment. As part of the enrolment process the RA also performs attestation of the device endpoint. As was highlighted in Section 2.3.1, the details of platform attestation are out of scope for this paper - we assume that the TPM has an EK for which an associated platform certificate exists. Through this certificate, the RA can, with standard TPM 2.0 functionality, assert the platform dependence of the enrolled eID authentication keys. In our architecture we also associate a number of remote authorizations for the TPM EA policy as being produced by the RA. Some of these authorizations are permanent, others need to be dynamically refreshed. In a production system this off-device Trusted Third Party (TTP) that produces the authorizations can be distributed, and need not necessarily always be associated with the RA function, but it nevertheless is an integral part of the security of our design.

The Certification Authority (CA) is the entity that provides the user certificate for the eID key as part of the enrolment process. It is common practice that the $\mathrm{CA}$ is not combined with the RA, and often the CA is physically isolated from the network used by the other entities in the system.

The Service Provider (SP) is the entity that leverages the eID, by validating the certificate and a signature by the eID key on a fresh challenge provided by the SP. A very typical use case in this context is a client-authenticated TLS sessions to a government service or a bank ${ }^{13}$.

The REE helper is an important component in our design, although not many security requirements are put on it. Since the TPM interfaces, and especially policy generation are quite different from what can be expected by ordinary developers mostly interested in making his/her application eID-aware, the REE helper provides the interface typically used for ID access and use - the PKCS\#11 standard, and operates to fulfill the requirements identified in Section 3. The principal function of the REE helper is therefore to translate the eID APIs and requirements into TPM library commands and concepts. Since the usage policy in our example will be mapped to TPM EA policy, we do not have to require perfect integrity of the REE helper. In case its integrity is compromised, the main possible consequence is that the TPM policies will not be satisfied, leading to a $D e-$ nial of Service (DoS) situation.

\subsection{Authorization Policy}

We discussed the basics of the TPM 2.0 EA policies in Section 2.3.4. In this section we will describe how to to express the following aspects of our requirements using EA policy primitives:

- Platform integrity

(Section 4.2.1)

- Restricting the number of PIN attempts (Section 4.2.2)

- Reset of the PIN entry counter

(Section 4.2.3)

- PIN entry and comparison

(Section 4.2.4)

- Credential revocation (expiration)

(Section 4.2.5)

Figure $3 \mathrm{~b}$ illustrates the TPM parameters and objects we will use in the following description. Each following section is accompanied by a listing of the TPM commands invoked by the REE-helper to satisfy the policy. In these listings, each command is represented in the form:

$[$ digest $\leftarrow]$ command $(<$ in parameters $>[,<$ out parameters $>])$

\subsubsection{Platform integrity}

13 eID is also often used for document signatures, in which case the role of the SP becomes slighly different. 


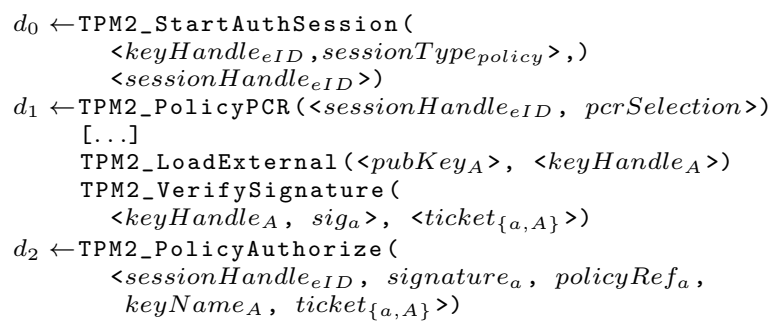

Listing 1: Platform integrity policy fragment

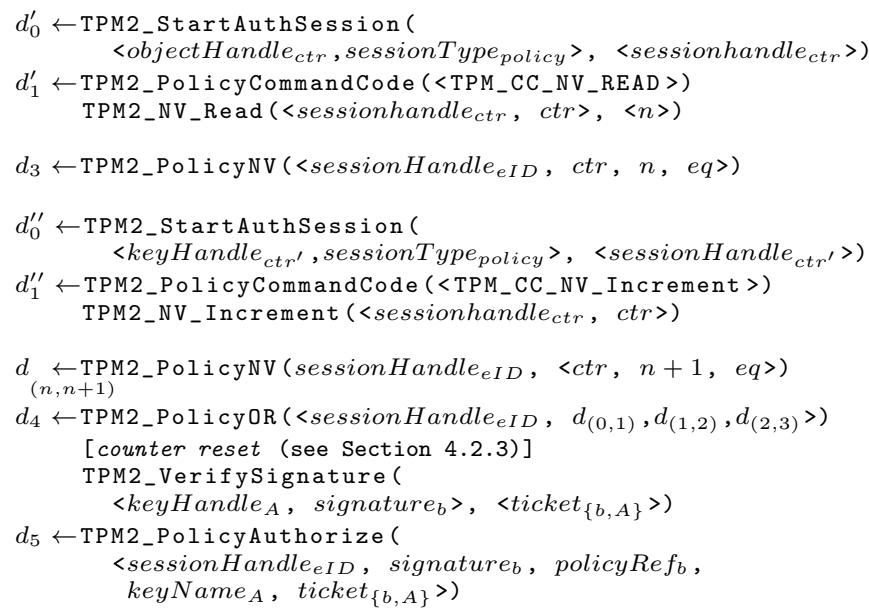

Listing 2: PIN attempt restriction policy fragment

The baseline of the policy (see Listing 1) is the invocation of one or more TPM2_PolicyPCR() commands. The PCR registers are reliably populated by boot-up measurements by the CRTM, and these PCR values cannot be re-populated to any known values after boot. Therefore, a TPM2_PolicyPCR command that maps the values of these PCRs into the policy session will identify whether the device was booted into a software state recognizable as part of the policy, or into some other state.

To make the policy modular, e.g. to make it possible to update the REE helper to a newer version, it makes sense to partition the policy collection with external authorizations. In Listing 1 , this is based on an external public key which is loaded to the TPM using the TPM2_LoadExternal() command. Now, the external authorization can occur as described in Section 2.3.4; first, the signature of the external authorization, which is of a standardized format, is validated with TPM2_VerifySignature(); second the locally signed ticket produced by TPM2_VerifySignature() is passed to TPM2_PolicyAuthorize(). As we can recall from our earlier example, if the validated authorization matched the current policyDigest for the session, the policyDigest is reset, and replaced, essentially, by the public key hash of the public key used to verify the signature.

\subsubsection{Restricting the number of PIN attempts}

The next policy aspect we consider is the number of failed PIN attempts for the key we intend to use (Req 1(a)ii). To capture this we use a NV storage element configured to act as a counter, i.e. during its initialization it is associated with an TPMA_NV_COUNTER attribute. This makes writes to the NV element only possible in a monotonically increasing manner using the TPM2_NV_Increment () command. We also assume that the NV object holding the counter is associated with an authorization policy of its own, where resetting the counter is conditional to the entering of the correct PIN value as part of the policy, but incrementing the counter is allowed for the REE helper without restriction. The specifics of this policy are described in Section 4.2.3. For now, the goal is to bind proof of increment (that the counter has been incremented by one during the policy session) into the policyDigest, and assert that the final counter value is less than, or equal to the allowed number of PIN attempts (see Listing 2). Note that the invocation of TPM2_NV_Increment () cannot be directly determined in the policy session for the eID credential. Hence a mere comparison between the current counter value and the allowed number of attempts is insufficient to ensure proper operation. Instead, we use TPM2_NV_Read() to retrieve the current counter value in the REE helper. Then we bind the current counter value to the policy digest of the main policy session using TPM2_PolicyNV(), increment the counter in a separate session, then proceed to bind the now incremented counter value to the main session policy digest. Recall from Section 2.3.4 that TPM2_PolicyNV() makes its comparison immediately, not in a deferred manner.

Let us assume that the maximum number of (failed) PIN entry attempts allowed is three. In this case, the previous policy segment should accept combinations of the NV storage element value pairs $(0,1),(1,2)$ and $(2,3)$. All of these represent different acceptable policyDigest values for the policy session. The values can be collapsed into one using the TPM2_PolicyOR() conjunction (see Section 2.3.4). The REE helper, possibly with the aid of the RA, can compute these expected values, which each is the result of the consecutive application of the prior TPM2_PolicyAuthorize() and two TPM2_PolicyNV() invocations for one of the above pairs. The resulting policyDigest value is now independent of the value pair that was encoded into the policyDigest prior to the TPM2_PolicyOR().

For consistency, we again normalize the policy construction by the application of another external authorization originating from the RA. The reasoning for this is similar to the first stage normalization - for the PIN count we can envision variations in e.g. which NV storage element is used as the PIN counter, or possibly the maximum allowed attempts. Note that signature used for this normalization should include a different policyRef from the one applied before. This is needed so that the TPM implicitly protects against policy fragment re-ordering or the omission of some fragments in the middle of a policy session.

\subsubsection{PIN counter reset}

On each attempted use of the eID signature key, the PIN entry counter is incremented. If the use of the signature key succeeded, we know that a) the PIN received at the REE helper was correct, and b) that the counter value had not passed the threshold for allowed tries. If the threshold was exceeded, we require that a PUK value is entered correctly for the PIN counter to be reset. For this policy, we formalize the PUK as high-entropy system-generated password value that requires no inherent replay protection. However, as shown by the PIN handling in the main policy, the PUK could equally well be modelled like the PIN, with the same replay-protection setup. 
The TPM 2.0 specification does not provide the possibility to reset a counter $\mathrm{NV}$ object, i.e. the actual reset operation must be split into two parts; the erasure of the counter object (to which an associated policy is applied), and its recreation. The object recreation is conditioned to "platform-specific" user authorization, which may be vary from completely open to any platform-issued policy, i.e. as a worst case we must assume it to be completely unprotected. Herein lies one threat against our system for PIN replay protection. As the counter reset is not atomic, if an attacker can disrupt the object creation e.g. power-cycle the device with a physical reset switch at exactly the right moment after a successful authentication, the attacker might be able to recreate the counter with a policy that allows reset with less privileges. This sets the stage for brute-force PIN resolving, which in turn may lead to unauthorized use of the signing key. This also constitutes one reason for why the integrity measurement of the REE helper (see Section 4.2.1) matters in the overall policy. Considering the complexity of the attack, we argue that an attacker of this strength may do better by just eavesdropping the PIN value when entered by the user. There are also processes for command audits in the TPM 2.0 specification that may be applied as protection for the atomicity problem, but these require another internal or external trusted environment for validation.

The initial phase of the policy construction for PIN counter reset mirrors that of key usage. First, the integrity of the system and the REE helper is assured, followed by the external authorization, as outlined in Section 4.2.1. The second phase has two alternative paths. The first one mirrors the updating of the PIN retry counts but sets the upper limit to $n+1$, i.e. if the limit was 3 , as in the earlier example, we use 4 as the limit here. This is followed by the entering of the PIN, described in Section 4.2.4. The second path is the PUK entry option. Here, the initial value composition also include the integrity checks for the system and REE helper. This is followed by binding the preferably strong PUK password to the policy by the application of a TPM2_PolicySecret() in a manner equivalent to PIN entry (see Section 4.2.4.

The two possible policySession values are then combined using TPM2_PolicyOR(), possibly followed by a TPM2_PolicyAuthorize() for good measure. The final step of the policy will have to account for the current state of the session augmented with TPM2_PolicyCommandCode() for the command TPM2_NV_UndefineSpace. The resulting session value must be ORed with the completely open policy for counter updates and reads, i.e. a policy with TPM2_PolicyCommandCode() for the commands TPM2_NV_Increment or TPM2_NV_Read. Finishing up the object authPolicy value after this follows the main policy for key use.

PIN updates also follow from the right to remove the NV object holding the PIN. As defined in this case, those objects have secret-based authorization, i.e. erasing them requires only the knowledge of the respective PIN or PUK. The recreation of the NV record, i.e. adding a new PIN with TPM2_NV_DefineSpace() may require user authorization (a user password).

\subsubsection{PIN entry}

The next stage of the policy after PIN counter handling is the entry of the PIN. There are two dedicated pol-

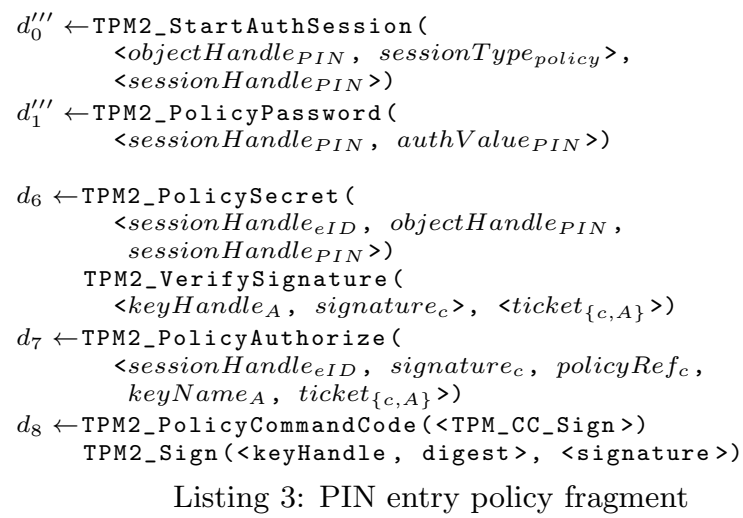

Listing 3: PIN entry policy fragment

icy commands for this, the TPM2_PolicyAuthValue() and TPM2_PolicyPassword() both of which, with slight variations, defer a mandatory check of the PIN for the authorized key object before object use (recall that most TPM 2.0 objects can contain a password value for authorization, this is a feature that is inherited from the TPM 1.2 specification). However, associating the PIN value with the key object is not acceptable, as we want to separate PIN objects from keys to allow a particular PIN to be shared by many keys (Req. 1(a)i, and manage the life cycle of these PINs (e.g. change of the PIN value) in isolation from the eID key objects referring to them. The TPM 2.0 specification provides a policy command ideally suited for this kind of setup: TPM2_PolicySecret() lets us associate the knowledge of a secret, encoded in another object's authValue with the policy of the object we are intending to use. The policy digest will be updated with the name of the object we associating to. In principle we could associate to any kind of object, e.g. a "dummy" key, but using a NV record for this purpose has the advantage that its name does not change when re-created, whereas the public key component of an asymmetric key pair is represented in its name. Thus we use an empty NV record with the PIN as its authValue as the associated object. On correct PIN entry (see Listing 3) the REE helper is empowered with the knowledge necessary to both validate, erase and replace the NV object with the $\mathrm{PIN}$, and it is up to the integrity protection of the REE helper to ascertain its correct operation with regards to using (and erasing the PIN from its memory) in the different TPM operations involving PINs and PUKs ${ }^{14}$. This part of the policy may once again be rounded off with the application of TPM2_PolicyAuthorize(), to potentially allow e.g. for device-specific NV index variations for PIN handling.

Finally, TPM2_PolicyCommandCode() (for TPM2_Sign()) is invoked to limit the capabilities the authorization sessions grants on the key object. The final session policy value (which is an aggregation of the device-specific license authorization (public key) and the command code constraint) is the value that shall be added to the key object when the key is created by TPM2_Create(). The value can be attested by the RA, since key creation can happen in a remoteoriginated authenticated channel, and the value can be confirmed by returned attributes (e.g. creationData). In fact,

\footnotetext{
${ }^{14}$ The obvious vulnerabilities with PIN handling in the REE can be remedied by hardware-supported secure PIN entry. This "Secure UI" can be mapped to the TPM concept of a higher locality - a binding that can be included in TPM EA policy. Such setups are for now highly device-specific, and we do no consider this further in this paper.
} 
if the RA makes sure that no two licenses issued to a specific endpoint are alike, all keys can be created with the same authPolicy value. The policy construct is flexible enough to account for all optionality as part of the individual RAoriginating authorizations. This even holds when the policy is augmented with extra functionality, e.g. such as described in the following section.

\subsubsection{Credential revocation}

With removable tokens such as smart cards, credential revocation often relies solely on certificate revocation, and elaborate protocols and constructs such as Certificate Revocation Lists (CRLs) and on-line protocols such as the OnLine Certificate Status Protocol (OCSP) allow for service providers to confirm the revocation status of a credential within the lifetime indicated in its certificate. However, with TEEs and TPMs, credential revocation can also be managed at the client-side as an additional security measure. The TPM 2.0 specification has a millisecond-resolution clock that is guaranteed to advance when the TPM is powered on, but it also has periodic backup to NV memory, i.e. within certain tolerance it behaves like a secure clock. Policy access to this clock is through the TPM2_PolicyCounterTimer() policy command. The command behaves as a timer, i.e. a time comparison is made based on "time passed" since a reference value which is provided in the command parameters, and which also is reflected in the policy value update. This can be straight-forwardly mapped to a subsequent TPM2_PolicyAuthorize() with a device-specific key originating from the RA, i.e. the RA can this way provide time-dependent licenses for the usage of the eID key that are more short-lived than the CA-generated certificate - a "dead man's switch" of sorts. This approach makes practical sense, since it eases the interactive load on service providers, who now can trust that signatures against eID certificates residing in TPMs (a fact that can be logged in an certificate attibute extension) have this "automatic" revocation property, i.e. the keys stop being usable if key revocation occurs within the time period of the CA-issued certificate.

\section{ANALYSIS}

We limit the scope of our analysis to the presented eID requirements in the context of constructs described in Section 4.2. While we acknowledge that formal verification of the TPM 2.0 EA primitives is a worthy pursuit, we consider it out of scope for this paper. Likewise, as all TPM 2.0 platforms are by definition required to provide platform assurance, it is not in our power to add to or detract from the trustworthiness of the platform itself.

In terms of functional requirements, the EA policy binds key authorization to any number of shared or disjunct PINs and PUKs, and as the TPM by internal design can support any number of key objects, we claim that requirements by both EU eID and FIDO are easily met. Necessary security guarantees are provided by the TPM 2.0 itself. Of the privacy requirements, we do address linkability during token use. There may also be linkability concerns during enrolment and platform authentication, these are issues external to this paper, but TPM 2.0 does provide optional Direct Anonymous Attestation [3] (DAA) support to mitigate even this threat. For credential user binding, we leverage TPM $2.0 \mathrm{EA}$, and provide the example as proof that we can provide retry-protection akin to that of PKCS\#15 tokens.
Furthermore, we provide the dead man's switch - type behaviour for the eID credential. This partially offline system is one way of allowing eID to be reliably used with SPs that have only point-to-point connectivity with the ESP, such as physical doors or out-door event ticket validators.

We also want to highlight the relaxed integrity requirements on the REE helper as an important side-effect of leveraging TPM 2.0 EA. Even as the presented authorization model involves a large number of commands and operations, the entities securing the authorization are the policy sessions, in turn proteced by TPM 2.0. Except for the atomicity issue highlighted in Section 4, all operational misbehaviour from the side of the REE helper constitutes only Denial-of-Service.

\section{PERCEIVED EA SHORTCOMINGS}

Despite the flexibility of the TPM 2.0 EA mechanism, on several occasions we find the need to resort to awkward constructs in order to formulate primitives needed for our policy. We identify the following instances where alternative design decisions than the ones made by the TPM 2.0 designers could have made policy constructions simpler and more intuitive:

Including a secret value as part of a policy The current TPM 2.0 specification EA policy command set does not allow a secret value to be included as part of the policy. A password entry, for instance, could be trivially turned into a policy command, hashing that password directly into the session policy value. Such a command could have found immediate use in our design, allowing us to avoid the use of "dummy" NV objects in Section 4.2.4 to hold the PIN and PUK.

Linking two consecutive TPM commands The current specification does not allow a TPM command to be conditional on the outcome of another. For example, with such a feature, we could have specified a policy: "reset PIN counter only after successful sign-operation" which could have made the PIN counter reset detailed in Section 4.2.3 simpler, and thus stronger.

Access rights for comparing TPM objects Another shortcoming that we encountered, is that comparisons between TPM objects, especially NV elements, can only be done while obtaining read rights to the object being referred to. This makes sense from a data-flow standpoint, but makes many policy constructs cumbersome or even impossible.

\section{RELATED WORK}

The legal, technical and organizational challenges in implementing a pan-European eID framework have been analyzed in a number of studies. The study by Myhr [13] mainly focuses on the issuance procedures and the lack of a common unique identifier for physical persons on an European level that could be used in eID. Since the publication of the study, a large study was conducted by the European Union regarding eID interoperability for Pan-European EGovernment Services (PEGS) ${ }^{15}$. The results of this study are a final report consisting of analysis and assessment of eID interoperability requirements and 32 distinct country profiles on national schemes from both a legal and technical perspective. Mahler [11] in turn proposes a multi-stakeholder governance model for an European eID influenced by multi- 
stakeholder institutions used in Internet governance, such as the ICANN and IETF.

There are also a few technical studies with the aim of overcoming some of the limitations and challenges with conventional, smart card-based identity tokens. TPMident [6] is a two-factor authentication system based on TPMs, with the goal of protecting users against identity theft. In TPMident, conventional eID is used to establish initial trust to a non-migratable authentication credential stored within a TPM. The authors have also integrated TPMident with the OpenID ${ }^{16}$ sign-on protocol.

Dmitrienko et al. [4] present the design of a token-based access control system for NFC-enabled smartphones which allows users to maintain access control credentials for multiple resources. A key feature of their scheme is the ability delegate access rights to other smartphone users without the involvement of a central authority (RA).

Vossaert et al. [18] propose a system for secure PINentry for smart cards. The solution relies on a workstation equipped with a TEE and an attached smart card reader. The authors present a proof-of-concept prototype of the system using Belgian eID cards and PCs with TPM-based secure execution environments.

\section{CONCLUSIONS}

On one hand, our eID architecture demonstrates that the new TPM 2.0 Enhanced Authorization model can meet the requirements of a widely deployed, real-world use-case. On the other, despite the richness of the current EA model, we identify some possible improvements that would enable simpler, and thus more secure, solutions. We believe that adding TPM 2.0 to the set of possible ESP implementations will add a large class of (mobile) devices as eID endpoints.

In Section 5 we identified some shortcomings of the EA model that, if addressed in TPM specifications, can increase the flexibility of TPM 2.0 EA even further. Even with such improvements, we still have to recognize the difference between full token programmability in an isolated, secure environment and a configurable authorization model to a well defined and analyzed set of security primitives. Both approaches have their individual merits on the axes of flexibility vs. security.

As future work, we plan to complete a working Proofof-Concept ESP as presented in this paper, to be trialed against a government PKI. Other research avenues include integrating biometrics and other trustworthy I/O to the ESP design.

\section{ACKNOWLEDGMENTS}

This work was financially supported in part by the Intel Collaborative Research Institute for Secure Computing and by the Academy of Finland, project No 283135 (CloSe: Cloud Security Services).

\section{REFERENCES}

[1] A New Model: The Consumer-Centric Model and How It Applies to the Mobile Ecosystem. Tech. rep., GlobalPlatform, Mar. 2012.

\footnotetext{
${ }^{15} \mathrm{http}$ ///ec.europa.eu/idabc/en/document/6484.html

${ }^{16}$ http://openid.net/
}

[2] Andrade, N., Monteleone, S., and Martin, A. Electronic Identity in Europe: Legal challenges and future perspectives. JRC-IPTS Working Papers JRC78200, Institute for Prospective and Technological Studies, Joint Research Centre, Dec. 2013.

[3] Chen, L., AND Li, J. Flexible and Scalable Digital Signatures in TPM 2.0. In Proceedings of the 2013 ACM SIGSAC Conference on Computer E\#38; Communications Security (New York, NY, USA, 2013), CCS '13, ACM, pp. 37-48.

[4] Dmitrienko, A., Sadeghi, A.-R., Tamrakar, S., and Wachsmann, C. SmartTokens: Delegable Access Control with NFC-Enabled Smartphones. In Proceedings of the 5th International Conference on Trust and Trustworthy Computing (Berlin, Heidelberg, 2012), TRUST'12, Springer-Verlag, pp. 219-238.

[5] Ekberg, J.-E., Kostiainen, K., And Asokan, N. The Untapped Potential of Trusted Execution Environments on Mobile Devices. Security Privacy, IEEE 12, 4 (July 2014), 29-37.

[6] Klenk, A., Kinkelin, H., Eunicke, C., And CArle, G. Preventing Identity Theft with Electronic Identity Cards and the Trusted Platform Module. In Proceedings of the Second European Workshop on System Security (New York, NY, USA, 2009), EUROSEC '09, ACM, pp. 44-51.

[7] Laitinen, P. Personal communication, Feb. 2014. Senior analyst, Finnish Population Register Centre.

[8] Lenstra, A. K., And Verheul, E. R. Selecting Cryptographic Key Sizes. Journal of Cryptology 14 (1999), 255-293.

[9] Lindeman, R., Baghdasaryan, D., And Hill, B. FIDO Technical Glossary. Specification Set: fido-v1.0-rd-20140209 REVIEW DRAFT. https: //fidoalliance.org/specs/fido-glossary-v1.0-rd-20140209.pdf, Feb. 2014.

[10] Lindemann, R. The Evolution of Authentication. In ISSE 2013 Securing Electronic Business Processes, H. Reimer, N. Pohlmann, and W. Schneider, Eds. Springer Fachmedien Wiesbaden, 2013, pp. 11-19.

[11] Mahler, T. Governance Models for Interoperable Electronic Identities. Journal of International Commercial Law and Technology 8, 2 (2013).

[12] Martens, T. Electronic identity management in Estonia between market and state governance. Identity in the Information Society 3, 1 (2010), 213-233.

[13] Myнr, T. Legal and Organizational Challenges and Solutions for Achieving a pan-European Electronic ID Solution. Inf. Secur. Tech. Rep. 13, 2 (May 2008), 76-82.

[14] Naumann, I., And Hogben, G. Privacy features of European eID card specifications. Network Security 2008, 8 (2008), 9 - 13 .

[15] Nyman, T., Ekberg, J.-E., And Asokan, N. Citizen Electronic Identities using TPM 2.0. Tech. rep., Aalto University, 2014, arXiv:1409.1023 [cs.CR].

[16] Trusted Computing Group. TPM Main Specification Level 2 Version 1.2, Revision 116. Parts 1-3. http://www.trustedcomputinggroup.org/resources/tpm_ main_specification, Mar. 2011.

[17] Trusted Computing Group. Trusted Platform Module Library Specification, Family "2.0", Level 00, 
Revision 00.99. Parts 1-4.

http://www.trustedcomputinggroup.org/resources/tpm_ library_specification, Oct. 2014.

[18] Vossaert, J., Lapon, J., De Decker, B., And NaEssens, V. Trusted Computing to Increase Security and Privacy in eID Authentication. In ICT Systems Security and Privacy Protection, N. Cuppens-Boulahia, F. Cuppens, S. Jajodia, A. Abou El Kalam, and T. Sans, Eds., vol. 428 of IFIP Advances in Information and Communication Technology. Springer Berlin Heidelberg, 2014, pp. 485-492.

[19] Wilkins, R., And Richardson, B. UEFI Secure Boot in Modern Computer Security Solutions. Tech. rep., UEFI Forum, Sept. 2013.

\section{APPENDIX}

\section{A. TPM 1.2 AUTHORIZATION PROTO- COLS}

The TPM 1.2 specification includes three protocols to securely pass proof-of-knowledge of the owner authorization value; the Object-Independent Authorization Protocol (OIAP), the Object-Specific Authorization Protocol (OSAP), and the Delegate-Specific Authorization Protocol (DSAP).

\section{OIAP}

The OIAP protocol supports multiple authorizations sessions to arbitrary entities; in other words, a single OSAP session can be used to invoke multiple commands on the TPM, which manipulate different objects. However, an OSAP session cannot be used to introduce new authorization information to the TPM (e.g. key creation), as the owner authorization value of the entity being accessed is used to key the Hash-based Message Authentication Code (HMAC) used for authentication and integrity verification of session traffic.

\section{OSAP}

The OSAP protocol supports authorization sessions for a single entity, i.e. all commands invoked during a particular OSAP session may only manipulate the same entity. The advantage is that OSAP enables the confidential transmission of new authorization information. OSAP session initialization involves the creation of a ephemeral secret (henceforth referred to as the OSAP shared secret) used to protect further protocol traffic. The purpose of this arrangement is two-fold; first, it allows the caller to cache the OSAP shared secret for the duration of an extended session without compromising the owner authorization value of the entity being accessed; second, it enables new authorization information to be inserted into the TPM. The insertion of new authorization data is covered by the AuthData Insertion Protocol (ADIP), which involves encryption of the new authorization data using a one-time key generated computing a hash of the OSAP session secret and a session nonce. A similar scheme is utilized by the AuthData Change Protocol (ADCP) to change the owner authorization associated with an existing entity. Knowledge of the old owner authorization value is required as a proof-of-ownership in order to change the authorization information of an entity. As reusing the OSAP shared secret multiple times could expose it to cryptoanalytic attacks, the TPM is responsible of terminating OSAP sessions that have introduced new authentication data with ADIP or ADCP. The caller is responsible for terminating any authorization session to ensure the removal of any saved authorization information and ephemeral secrets.

\section{DSAP}

The DSAP protocol allows access to an entity to be delegated without disclosing the owner authorization value to a third party. The owner of the key may dictate the set of commands that can be performed using the delegated cryptographic key when authorization other than actual owner authorization is used. For instance, the recipient of a delegated key may be allowed to use the key for encryption or decryption, but may not delegate the key further or modify the authorization information associated with the key. This is realized via the creation of a delegation structure by the key owner. The delegation structure specifies the cryptographic key to be delegated, the set of commands that may be performed using the key, and a delegation authorization value. The delegation structure itself is encrypted with a key known only to the TPM. The purpose of this is to protect the confidentiality of the delegation authorization value associated with the delegation structure, as any caller who can supply the structure, and has knowledge of the associated delegation authorization value, may use the delegation structure to perform the operations specified by the structure on the delegated key. This allows the delegation mechanism to operate without changes to TPM commands which expect a authorization value to be specified upon invocation.

Similarly to OSAP session, DSAP protocol session are restricted to a single entity. During DSAP session initialization, the caller specifies the target entity, and supplies the delegation structure for that object. Also similarly to OSAP, the DSAP session initialization involves the creation of an ephemeral secret used to protect further traffic. During the DSAP authorization session, the caller may invoke any commands allowed by the specified delegation structure by supplying the delegation authorization value of that structure instead of the owner authorization value associated with the object being accessed.

\section{B. TPM 2.0 EXTENDED AUTHORIZA- TION COMMANDS}

This section provides a brief overview of the Extended Authorization commands referred to elsewhere in this paper.

\section{B.1 TPM2_PolicyAuthorize()}

The TPM2_PolicyAuthorize() command authorizes changes to an existing policy by an external entity. The authorizing entity signs the digest value it wants to authorize. The TPM will verify the signature, and if valid, the TPM2_PolicyAuthorize() will reset the current policyDigest value and update it with a digest derived from the (unique) name of the signing key:

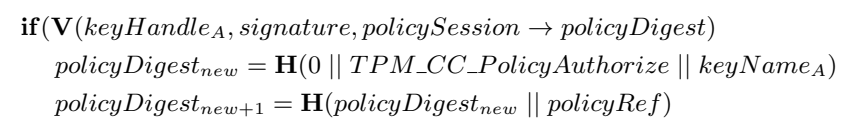
where

TPM_CC_PolicyAuthorize is the policy command code A is the authorization key $\mathbf{V}()$ is the signature verification algorithm for $A$ 
keyHandle $_{A}$

signature

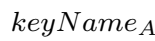

policyRef is the handle for $A$

is the signature of the policy to be authorized

is the name of the object corresponding to $A$

is the policy classifier
External authorization is discussed in more detail in Section 2.3.4.

\section{B.2 TPM2_PolicyAuthValue()}

The TPM2_PolicyAuthValue() command is a deferred assertion that sets the policySession $\rightarrow$ isAuthValueNeeded property. This indicates a caller provided authValue will be compared the authValue of the authorized object when the policy session is used for authorization. This property also indicates that the caller provided atuhValue is included in the key used to calculate the authorization HMAC for the current session. This key is provided as parameter to TPM2_BeginAUthSession. Once set, the isAuthValueNeeded property may only be unset by TPM2_PolicyPassword() or TPM2_PolicyRestart(), which returns the entire authorization session to its initial state. The policyDigest derived by TPM2_PolicyAuthValue simple binds the corresponding command code as part of the running digest:

policyDigest $_{n e w}=\mathbf{H}\left(\right.$ policyDigest $_{\text {old }} \|$ TPM_CC_PolicyAuthValue $)$

\section{B.3 TPM2_PolicyCommandCode()}

TPM2_PolicyCommandCode() command is a deferred assertion that verifies that the policy session is only used to authorize a particular command. The command code for the authorized command is stored in policySession $\rightarrow$ commandCode property. When the session is used for authorization, the stored command code is compared to the code of the invoked command. The stored command code is also bound to the policyDigest:

policyDigest $_{\text {new }}=\mathbf{H}\left(\right.$ policyDigest $_{\text {old }} \|$ TPM_CC_PolicyCommandCode $\|$ code $)$

where

TPM_CC_PolicyCommandCode is the policy command code code is the code for command code being authorized

\section{B.4 TPM2_PolicyNV()}

The TPM2_PolicyNV is an immediate assertion, which performs an arithmetic comparison between an input value and a value stored in a specified non-volatile storage element. If the condition holds, the policyDigest is updated accordingly:

if $N$ Value op operand then

policyDigest $_{\text {new }}=\mathbf{H}\left(\right.$ policyDigest $_{\text {old }} \|$ TPM_CC_PolicyNV $\|$ args $\|$ NV Name $)$ where

TPM_CC_PolicyNV is the policy command code args is a hash over the input operands and operator NVValue is the value of the non-volatile storage element NVName is the name of the non-volatile storage element

\section{B.5 TPM2_PolicyOR()}

The TPM2_PolicyOR() command authorizes one of several possible digest values. It leads to an immediate assertion which verifies if the current policyDigest value occurs in a list of authorized digest values provided as input. If this is the case, the policyDigest is reset, and updated to a value derived by calculating a digest of the concatenated input digest values:

$$
\begin{aligned}
& \text { if }{\text { policySession } \rightarrow \text { policyDigest in } \text { digest }_{1} \ldots \text { digest }_{n} \text { then }}^{\text {policyDigest }} \text { new }_{\text {new }}=\mathbf{H}(0 \| \text { TPM_CC_PolicyOR } \| \text { digests }) \\
& \text { where }
\end{aligned}
$$

TPM_CC_PolicyOR is the policy command code digest $_{1} \ldots$ digest $_{n} \quad$ is a list of valid digest values ${ }^{17}$ digests $\quad$ is the concatenation digest $_{1}\|\ldots\|$ digest $_{n}$

\section{B.6 TPM2_PolicyPassword()}

The TPM2_PolicyPassword() is a deferred assertion that sets the policySession $\rightarrow$ isPasswordNeeded property. This indicates a caller provided authValue will be compared the authValue of the authorized object when the policy session is used for authorization. Once set, this property may only be unset by TPM2_PolicyAuthValue() or TPM2_PolicyRestart().

The TPM2_PolicyPassword differs from TPM2_PolicyAuthValue in that the authValues are compared as a clear-text passwords, not HMACs. In other respects, the commands are identical to the point were they actually use the same policy command code in the policyDigest update:

policyDigest $_{n e w}=\mathbf{H}\left(\right.$ policyDigest $_{\text {old }} \|$ TPM_CC_PolicyAuthValue $)$

\section{B.7 TPM2_PolicyPCR()}

The TPM2_PolicyPCR() command asserts the state of a specified set of PCRs, and can act as a combined or deferred assertion depending on if the caller provides and expected value for the PCR measurements, or not. If the caller provides an expected value, the value of the specified PCR is compared immediately to the expected value, else the validity of the PCR values will not be known until the policy session is used for authorization. Either way, the policyDigest is updated accordingly:

policyDigest $_{n e w}=\mathbf{H}\left(\right.$ policyDigest $_{\text {old }} \|$ TPM_CC_PolicyPCR $\|$ PCRs $\|$ digest $)$

where

TPM_CC_PolicyPCR is the policy command code pcrs

digest is the bit mask of the PCR selection

To detect changes to PCRs after the TPM2_PolicyPCR() command has been invoked, the TPM keeps track of PCR changes by incrementing a monotonically increasing policySession $\rightarrow$ pcrUpdateCounter each time a PCR is updated. When TPM2_PolicyPCR() is invoked, and the immediate PCR value check succeeds, the current value of the PCR update counter is stored in the current session context. On subsequent TPM2_PolicyPCR invocations, and when the policy session is used for authorization, the value of the PCR update counter is compared against the stored counter; the authorization will fail unless the counter values match. 